\title{
Searching for MACHOs with Microlensing
}

\author{
C. Alcock
}

This paper was prepared for submittal to the

"Unsolved Problems"

Princeton, New Jersey

April 17, 1996

April 1, 1996

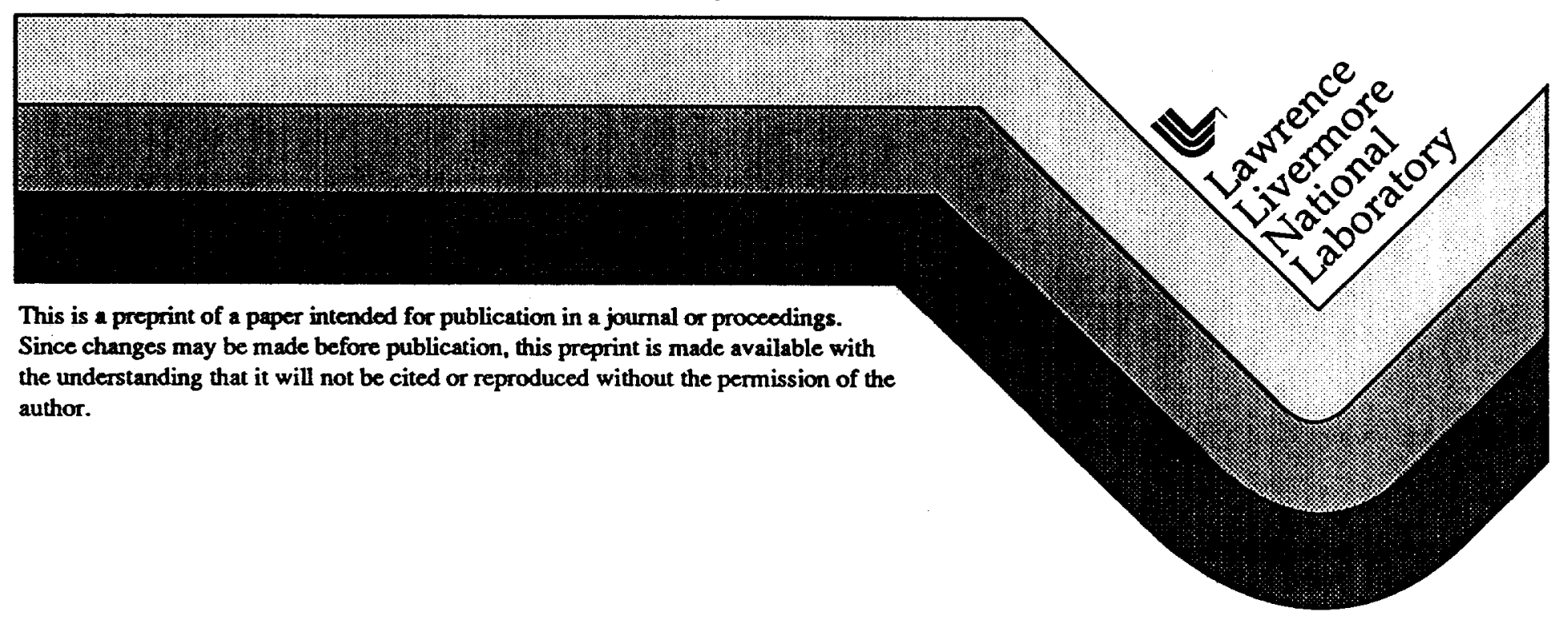




\section{DISCLAIMER}

This document was prepared as an account of work sponsored by an agency of the United States Government. Neither the United States Government nor the University of California nor any of their employees, makes any warranty, express or implied, or assumes any legal liability or responsibility for the accuracy, completeness, or usefulness of any information, apparatus, product, or process

disclosed, or represents that its use would not infringe privately owned rights. Reference herein to any specific commercial product, process, or service by trade name, trademark, manufacturer, or otherwise, does not necessarily constitute or imply its endorsement, recommendation, or favoring by the United States Government or the University of California. The views and opinions of authors expressed herein do not necessarily state or reflect those of the United States Government or the University of California, and shall not be used for advertising or product endorsement purposes. 


\title{
Searching for MACHOs with Microlensing
}

\author{
Charles Alcock \\ Lawrence Livermore National Laboratory, Livermore, CA 94550
}

June 2, 1996

\section{Abstract}

Baryonic matter, in the form of Machos (Massive Compact Halo Objects), might be a significant constituent of the dark matter that dominates the Milky Way. This article describes the experimental searches for Machos that exploit the gravitational microlens magnification of extragalactic stars. These surveys monitor millions of stars, in some cases every night, looking for magnification events.

The early results from the surveys have yielded some spectacular events, and pose a significant new puzzle for galactic structure: toward the Large Magellanic Cloud we see fewer events than anticipated for a standard dark halo dominated by Machos, but toward the galactic bulge, the event rate is much higher than anticipated.

This is a field of research that is ripe with opportunities for beginning (and senior) scientists. 


\section{Introduction}

Most of the mass of the Milky Way and similar galaxies is in some presently invisible form (see, e.g., the review of Fich and Tremaine [12]). This "dark matter" cannot be in the form of normal stars or gas, which can readily be detected. Additionally, there is compelling evidence for much larger quantities of dark matter on larger scales in the universe.

Many candidates have been proposed to account for this dark matter. These fall into two main classes: the particle-physics candidates such as massive neutrinos, axions or other weakly interacting massive particles (WIMPs) [24], and the astrophysical candidates, including substellar objects below the hydrogen burning threshold $\approx 0.08 \mathrm{M}_{\odot}$ ('brown dwarfs'), or stellar remnants such as white dwarfs, neutron stars or black holes; these are generically known as massive compact halo objects (Machos). Such objects would be much too faint to have been detected in current sky surveys.

The searches for Machos described here (and all of the experimental searches for particle physics candidates) are looking exclusively for objects located in the dark halo of the Milky Way. The total amount of dark matter in all galactic halos (located say, within $50 \mathrm{kpc}$ of spiral galaxies) is approximately known from rotation curve data, and contributes to the mean density of the universe $\Omega \sim 0.05$ : if $\Omega=1$, either the halos must extend far beyond $50 \mathrm{kpc}$ or there must be intergalactic dark matter.

Paczynski [21] suggested that Machos could be detected by their grav- 
itational 'microlensing' of background stars. This indirect technique does not depend upon light emitted by the Machos. (It should be remembered, though, that the lensing objects in question are not required to be dark, merely significantly fainter than the source stars.)

\section{The Gravitational Microlens}

The simple gravitational lens comprises a point-like source of light (typically a star), a point-like massive deflector (the Macho), and an observer. If the target star lies directly behind the Macho, its image will be a ring of light of angular radius, $\theta_{E}$, called the Einstein radius. If the Macho is separated from the line of sight to the source by some finite angle, $\theta=b / D_{\mathrm{d}}$ (where $b$ is the physical distance of the Macho from the line of sight), the ring splits into two arcs. The combined light from the two images produced by the gravitational lens causes a net magnification

$$
A(u)=\left(u^{2}+2\right) / u \sqrt{u^{2}+4}
$$

that depends only on the ratio of the angular separation to the Einstein radius, $u=\theta / \theta_{E}$. Note that $A>1.34$ when $u<1$, and $A \sim u^{-1}$ when $u \ll 1$. The Einstein radius is related to the underlying physical parameters by

$$
\theta_{E}=\sqrt{\frac{4 G M D_{\mathrm{ds}}}{c^{2} D_{\mathrm{d}} D_{\mathrm{s}}}}
$$


where $M$ is the Macho mass, and $D_{\mathrm{d}}, D_{\mathrm{s}}$, and $D_{\mathrm{ds}}$ are the (observer-lens), (observer-source), and (lens-source) distances, respectively. The term 'microlensing' is used when $\theta_{E}$ is so small that the two images cannot be separated with current observing equipment, and the image doubling cannot be seen.

Frequently the related quantity $R_{E}=\theta_{E} D_{\mathrm{d}}$ is referred to as the radius of the Einstein ring, where $R_{E}$ is the physical size of the ring described above, measured at the location of the Macho. For a source distance of $50 \mathrm{kpc} \approx 10^{10} \mathrm{AU}$ and a deflector distance of $10 \mathrm{kpc}$, the Einstein radius is $R_{E} \approx 8 \sqrt{M / \mathrm{M}_{\odot}} \mathrm{AU}$. The coincidence between this scale and the orbit of the earth around the sun can be exploited in the study of microlenses, as will be described below.

The observable phenomenon, as the Macho moves at constant relative projected velocity $v$, is the varying magnification of the star. This magnification is given as a function of time by $A[u(t)]$, where

$$
u(t)=\sqrt{\omega^{2}\left(t-t_{0}\right)^{2}+\beta^{2}}
$$

and $\beta$ is the impact parameter in units of the Einstein radius, $t_{0}$ is the epoch of maximum magnification, and $\omega^{-1}(\omega \propto v)$ is the characteristic time (duration) of the event.

Of course many astronomical sources are variable. Fortunately, simple microlensing has distinctive signatures which can be used to discriminate it 
from intrinsic stellar variability:

- Since the probability that any given star will be significantly lensed is small, only one event should be seen in any given star (see discussion of microlensing optical depth).

- The deflection of light is wavelength-independent; hence, the star should not change color during the magnification.

- The events should have lightcurves well described by the theory (Eqs. 1 and 3).

All these characteristics are distinct from known types of intrinsic variable stars; most variable stars are periodic or semi-regular, and do not remain constant for long durations. They usually change temperature and hence color as they vary, and they usually have asymmetrical lightcurves with a rapid rise and slower fall.

In addition to these individual criteria, if many candidate microlensing events are detected, further statistical tests can be applied:

- The events should occur equally in stars of different colors and luminosities.

- The distribution of impact parameter $u_{\text {min }}$ should be uniform from 0 to the experimental cutoff $u\left(A_{\min }\right)$. 
- The event timescales and peak magnifications should be uncorrelated (after correcting for experimental selection effects).

When a microlensing event is detected and its light curve is measured, one determines three parameters, $\omega, t_{0}$, and $\beta$. Of these, only $\omega$ is related to the physical parameters of the lens:

$$
\omega=\frac{v}{D_{\mathrm{d}} \theta_{E}}
$$

For microlensing of sources in the Large Magellanic Cloud (LMC), $\omega^{-1} \approx$ $70 \sqrt{M / \mathrm{M}_{\odot}}$ days. One of the principal limitations of present experiments in this area, one which cannot be resolved with purely ground-based work, is that our uncertain knowledge of the quantities $D_{\mathrm{d}}, D_{\mathrm{ds}}$, and $v$ for an observed microlensing event means that the uncertainty in the inferred mass of the Macho, $M$, spans more than an order of magnitude [16]. The mass is our only clue to the true nature of the Macho.

Parallax, obtained by the simultaneous observation of a microlens event from telescopes separated by a distance of order $R_{E}$, allows one to measure a second parameter $[14]$,

$$
\tilde{v}=\left(D_{\mathrm{s}} / D_{\mathrm{ds}}\right) v
$$

(Generally one obtains one vector component of $\tilde{v}$, and the absolute magnitude of another.) 
The complete solution of a microlensing event requires, in addition, the determination of $\theta_{E}$. This is very difficult to achieve, since typically $\theta_{E}<$ $10^{-3}$ ". For a handful of cases in which the Macho passes directly in front of the source star (or nearly in front of the star), it will be possible to measure $\theta_{E}$. This is possible because the magnification equation given above must be modified to take into account the finite angular size $\theta_{S}$ of the star. The modification depends principally upon the ratio $\theta_{S} / \theta_{E}$. (An important complication is introduced by the center to limb variation of brightness over the face of the star.) If $\theta_{S}$ can be estimated spectroscopically, one can in turn infer $\theta_{E}$.

In the rare cases when one measures $\omega, \tilde{v}$, and $\theta_{E}$, the lens parameters mass, distance, and transverse velocity can each be determined. For example,

$$
M=\frac{c^{2} \tilde{v} \theta_{E}}{4 G \omega}
$$

\section{The "Macho Fraction" in the Galactic Halo}

The gravitational microlens 'optical depth' is the quantity that probes directly the Macho fraction of the dark matter, since it is 'proportional' to the density of microlensing objects along the line of sight to the target stars. If the distribution of the total mass density $\rho_{\text {total }}\left(D_{\mathrm{d}}\right)$ is known along a given line of sight, then the experimental estimate of the optical depth $\tau$ along this line of sight yields the fraction of the total dark matter that is in the form 
of Machos.

The 'optical depth' $\tau$ for gravitational microlensing is defined as the probability that a given star is lensed with $u<1(A>1.34)$ at any given time, and is

$$
\tau=\pi \int_{0}^{D_{\mathrm{s}}} \frac{\rho\left(D_{\mathrm{d}}\right)}{M} R_{E}^{2}\left(D_{\mathrm{d}}\right) d D_{\mathrm{d}}
$$

where $\rho$ is the density in Machos. Since $R_{E} \propto \sqrt{M}$, while for a given $\rho$ the number density of lenses $\propto M^{-1}$, the optical depth is independent of the individual Macho masses. Using the virial theorem, one finds that $\tau \sim$ $(V / c)^{2}$, where $V$ is the rotation speed of the Galaxy.

More detailed calculations [16] give an optical depth for lensing by Machos in the Galaxy of stars in the Large Magellanic Cloud of $\tau_{\text {LMC }} \approx 5 \times 10^{-7}$, under the assumptions that (1) all of the dark matter is in the form of Machos; and (2) the most naive model of the halo (spherically symmetric, small core radius) is correct. This very low value means that only one star in two million will be magnified by $A>1.34$ at any given time. (Note that this estimate assumes that all of the dark matter is in Machos, and hence is a crude upper limit to the optical depth.)

Surveys for gravitational microlensing follow millions of stars photometrically in order to obtain event rates of a few per year against the Large Magellanic Cloud. The optical depth in principle can be estimated directly from the experimental data, once a statistically significant number of events 
has been recorded. This interpretation is complicated, in practice, by inefficiencies introduced by the irregular sampling, and by the very crowded star fields that must be observed in order to make the large number of photometric measurements.

The interpretation of microlens optical depths in terms of the fraction of the dark matter in the form of Machos is limited by our poor knowledge of $\rho_{\text {total }}\left(D_{d}\right)$. The dark halo may be spherical or flattened [25], and if flattened it may or may not be aligned with the plane of the disk [13]. The core radius of the halo is not securely known. Also important is a complete understanding of the contribution of the disk and bulge to the total mass interior to the solar circle. This has turned out to be more significant than expected.

Improving our knowledge of $\rho_{\text {total }}$ is clearly important. Improvement can be obtained by measuring the optical depth along many well separated lines of sight, combined with the measured rotation curve of the disk. The variation of $\tau$ with location on the sky would provide us with a form of tomography of the dark matter distribution, which gives the missing shape information. The rotation curve data provide the normalization for $\rho_{\text {total }}$. To date measurements have only been attempted along two well separated lines of sight, towards the Large Magellanic Cloud and towards the central bulge of the Milky Way. The latter line of sight does not probe the dark halo unambiguously, but is very useful in probing the structure of the Milky Way, and thus helping to determine $\rho_{\text {total }}$. 


\section{The Experimental Situation}

Four groups have reported detections of microlens events: the MACHO Project $[3,4,5]$, the EROS Project [7], the OGLE Project [27, 28], and the DUO Project [1]. The MACHO Project follows $\sim 10^{7}$ stars in each of the Large Magellanic Cloud and the galactic bulge. The MACHO Project has recorded $\sim 90$ events, of which eight are toward the Large Magellanic Cloud, and the rest toward the galactic bulge. The EROS Project has followed $\sim 4 \times 10^{6}$ stars in the Large Magellanic Cloud, and recorded two events (both of these are possibly due to intrinsic stellar variation). The OGLE Project follows $\sim 2 \times 10^{6}$ stars in fields near the galactic center, and has recorded $\sim 18$ events, and the DUO Project follows $\sim 10^{6}$ stars also in fields near the galactic center, and has recorded $\sim 12$ events.

Two example events are shown in Figure 1. The upper panel shows a high magnification event from the MACHO Project (the star is in the galactic bulge). The lower panel shows an event that was noticed well before it reached maximum magnification. (The epoch at which the event was recognized is marked on the figure.) This "alert recognition" is a new capability now routinely used by both MACHO and OGLE. The MACHO Project currently uses the alert process to select events for more careful photometric monitoring at Cerro Tololo Interamerican Observatory, and for spectroscopic follow-up. Spectra have been obtained during two alert events, and in each

case spectra were taken at epochs spanning the maximum. The spectra 
showed no evolution during the events, confirming the gravitational lens interpretation.

The early results from these surveys are surprising. Preliminary estimates of the optical depth toward the Large Magellanic Cloud indicate that (1) the event rate is probably too high to be accounted for by a previously known population of objects; and (2) the event rate seems to be lower than what was expected for a spherical dark halo in which all of the dark matter is comprised of Machos [5]. The recent work of Bahcall et al. [8] has almost eliminated low luminosity stars as the objects responsible for the microlensing that is seen.

A quantitative examination of the MACHO observations towards the Large Magellanic Cloud [5] shows that the best-fit Macho fraction, assuming a standard model for the dark matter distribution, is $f \sim 0.2$, but with very large uncertainties. It is not possible to exclude the possibility that all of the dark matter is in the form of Machos (especially considering our poor knowledge of the true distribution of the dark matter), nor is it possible to exclude that essentially none of the halo dark matter is in Machos. These results do show that significant answers to the question of the Macho fraction are within reach of the current experiments, or by next generation experiments as described below.

The galactic bulge observations also have yielded substantial surprises. The estimated optical depths are $\sim 3 \times$ higher than the upper limits that 
were estimated prior to the experimental results $[29,4]$. (These upper limits $[17,22]$ were based upon standard models for the galaxy.) Suggestions have included (1) that the galactic bulge is a very massive bar, with the long axis pointing in our direction (a successful model fit to the OGLE and MACHO results using a pre-existing model bar was made by [30]); (2) that the mass of the disk is much greater than anticipated [4]; (3) that the dark halo has a low density or even hollow core [11]; and (4) that the dark halo is a very flattened structure, and the high rate toward the galactic center is due to halo Machos.

It is important to note that the interpretation of microlensing results toward the Large Magellanic Cloud is contingent upon understanding the galactic bulge results. In particular, the estimate of $\rho_{\text {total }}\left(D_{\mathrm{d}}\right)$ in the halo, which is combined with $\tau$ to obtain the estimate of the Macho fraction, is derived from the mass model of the Galaxy. The model of the bulge is an important component of the mass model.

\section{$5 \quad$ Next Generation Experiments}

The current experiments are far from completing their missions, and collectively they will clearly be able to detect and decipher many more microlensing events, probably tens of events toward the Large Magellanic Cloud and perhaps hundreds of events toward the Galactic bulge. It is probably more useful, however, to look at what can be achieved with more powerful or even 
substantially different experiments, based upon the experience of the current four projects.

\subsection{What can be achieved from the ground?}

The experience of the four groups allows us to project what could, in principle, be achieved from the ground. This assumes that next- generation experiments are exploited fully to realize the limits of ground-based microlens surveys.

It will be possible to measure the optical depth toward the Large Magellanic Cloud with reasonable accuracy.

It might be possible to do this also for the Small Magellanic Cloud. It will not be possible to obtain useful estimates of the microlens optical depth toward other targets that are high above the galactic plane. All other targets that contain adequate numbers of stars are either so distant that the observations are confusion limited (e.g., M33), or the stars are in globular clusters that are too faint to observe usefully from the ground. This limits the potential usefulness of the gravitational microlens tomography technique that was described above.

Two groups $([10,9])$ will attempt to measure microlens event rates toward M31. Their technique is sensitive only to Machos in the halo of M31, and will not in the end provide additional information about the nature and prevalence of Machos in our own galaxy.

Ground-based work alone will not be able to determine the Macho fraction 
of the dark matter. The uncertainties in the shape and radial profile of the dark halo will be the primary limitation. This limitation can be alleviated by using the Macho Parallax Effect, reducing the uncertainty about where along the lines of sight the individual Machos are, and adding some velocity information. This is discussed in the next subsection.

The situation with respect to the bulge observations is more promising. It is possible that ground-based observations will be able to distinguish between a large central bar, pointing in our direction, and a "maximal" disk (Han and Gould [18] have expressed doubts that this can be achieved). It will not be possible, however, to distinguish a "maximal" disk from an extremely flat halo; this would require either the parallax technique or the tomography technique.

Finally, there is an exciting new application of the gravitational microlens technique. It is possible to search for planetary companions around Machos by looking for the small distortions in the light curves that are produced [ 20 , 15]. This search would require a latitudinally spread network of telescopes providing nearly continuous monitoring of events that are found in a survey such as MACHO. Two groups are attempting to put such a network together: MACHO and PLANET [2].

\subsection{Observing Macho Parallax}

Earlier we showed the great value of measuring the projected velocity $\tilde{v}$, especially in elucidating the masses of Machos. This can be accomplished by 
the parallax technique: measuring an event from two different lines of sight [14]. From the ground one can measure Macho parallaxes only for events of exceptionally long duration, so that the acceleration of the earth around the sun significantly changes the projected velocity during the event. These long duration events are uncommon.

Figure 2 shows the light curve of one of the more spectacular events discovered by the MACHO project. This is the only event for which the effect of parallax motion has been detected [6]. There is a significant asymmetry in the lightcurve of this star, which is well fit when the motion of the earth is taken into account. The best fit indicates that the velocity of the Macho with respect to the line of sight between the source star and the Sun is $75 \pm 5$ $\mathrm{km} / \mathrm{sec}$ when projected to the position of the Sun. The knowledge of the projected velocity allows us to derive a relation between the mass of the Macho and its position along the line of sight as seen in Figure 3 (solid line).

Also shown on this Figure 3 are two likelihood estimates taken from [6]. The long-dashed line assumes a simple model for the velocity distributions of stars in the Galactic disk and bulge. The short-dashed line assumes additionally that the lensing object is a main sequence star, and that this star contributes the baseline flux observed (see [6] for details). From this figure, we can immediately conclude that the lens is either a dark object at $\sim 1.5 \mathrm{kpc}$ or a star at $\sim 2.5 \mathrm{kpc}$. If we had information like this for a large fraction of the microlensing events, then we would be able to tell whether the majority 
of the lensing objects are in the galactic disk, bulge, or bar. Similarly, toward the Large Magellanic Cloud, routine measurements of microlensing parallaxes would allow us to determine whether the observed events are Machos in the dark halo, the Milky Way disk, or perhaps in the Large Magellanic Cloud.

Parallaxes are difficult to measure from the ground; only for the longest duration events will the earth move far enough in its orbit during the event to make the parallax effect visible from the ground. This limits us to measurements made on events for which the Machos are in the galactic disk, and eliminates from useful study bulge and dark halo Machos (for which the events are of duration much shorter then six months, because of the high expected transverse velocities). For this vast majority of cases, the microlensing events will end before the earth has had a chance to move very far, so the parallax information will only be available if events can be observed simultaneously from the earth and a small satellite in a solar orbit of order $\sim 1$ AU away from the earth [14].

Satellite parallaxes would greatly advance the study of Machos. For events seen toward the Large Magellanic Cloud, measurement of $\tilde{v}$ would distinguish between Galactic Machos $\left(\tilde{v}<300 \mathrm{~km} \mathrm{~s}^{-1}\right)$ and those in the Large Magellanic Cloud $\left(\tilde{v} \sim 2000 \mathrm{~km} \mathrm{~s}^{-1}\right)$. For Galactic Machos, $\tilde{v} \sim v$, so that one could distinguish between Machos in the disk $\left(v \sim 50 \mathrm{~km} \mathrm{~s}^{-1}\right)$, the thick disk $\left(v \sim 100 \mathrm{~km} \mathrm{~s}^{-1}\right)$, and the halo $\left(v \sim 200 \mathrm{~km} \mathrm{~s}^{-1}\right)$. It would be possible to measure the transverse direction for at least some of these Machos, yielding 
additional information about their distribution.

The situation is somewhat more complicated for Machos seen towards the bulge. Parallaxes can statistically distinguish between Machos lying in the disk versus those which lie in the bulge itself, and also allow one to estimate the mass of the former [18]. Moreover, for $\sim 10 \%$ of bulge Machos and $\sim 4 \%$ of disk Macho events it should be possible to measure $\theta_{E}$ from the ground and therefore use the parallax to determine $M, D_{\mathrm{d}}$, and $v$ individually.

\section{Working on Gravitational Microlensing}

Research on gravitational microlensing has become a well established subfield of astrophysics, and there are abundant opportunities for young scientists. There is the special pleasure and reward that comes from working on a topic of great importance (in a period in which progress is being made rapidly), and there are few, if any, topics more important than determining what the universe (or at least, the Milky Way), is made of!

The experimental work differs in some important cultural ways from most contemporary astrophysics research. The most important difference is that the projects typically require teams that are much larger than the norm. (Some well-known astronomers have remarked to the author that they would not work in this area precisely for this reason!) A newcomer to this research should recognize that extremely demanding projects require larger than normal teams of scientists, and that group efforts are frequently necessary. In 
my view, the reward that comes from making an unusual contribution more than compensates for the reduced autonomy!

There is a notable exception to this remark: the DUO team is much smaller than the other successful microlensing survey teams. Keep in mind, though, that the only team that has repeatedly detected microlensing towards the Large Magellanic Clouds (MACHO) is relatively large.

Smaller groups and individuals dominate the theoretical and model building work which is needed to interpret the data and to suggest new directions. Prominent examples of this are the original paper in the field [21], and the many papers of Andy Gould.

I should make also a more general remark that comes from the history of the MACHO Project. There was great skepticism among our colleagues about this project, and it was not widely believed that we would be successful. We decided that the scientific goal more than justified the technical risks we were taking (and fortunately some key sponsors agreed), and went ahead in spite of the best advice of our friends. The outcome has convinced me that it is important to take one or more large risks in a career.

\section{Summary}

It is useful to summarize the situation in regard to research on gravitational microlensing in three parts: what we know, what we will likely learn from current experiments, and what we could learn with aggressive next generation 
experiments.

\subsection{What we know now:}

- Gravitational microlensing has been observed.

- The event rate toward the Large Magellanic Cloud is lower ( 20\% but with large errors) than expected for a "standard halo" filled with Machos of substellar mass.

- The event rate towards the galactic bulge is 3 times higher than expected.

\subsection{What we will learn from current experiments:}

- Reasonable statistics for the event rate towards the Large Magellanic Cloud (primary handle on halo Machos).

- Approximate event rate towards the Small Magellanic Cloud.

- Crude map of the event rate as a function of Galactic longitude and latitude near the galactic center (needed to disentangle galactic models).

\subsection{Next generation experiments:}

- Develop photometric follow-up network to determine lightcurves better (to measure higher order effects such as finite angular size of star, which 
yields much more information per event, and to search for planetary companions).

- Macho Parallax Satellite (measure lightcurves simultaneously from earth and spacecraft $1 \mathrm{AU}$ away to resolve the lens; this will determine where the Machos are).

\section{Late Breaking News}

Eight months after the Unsolved Problems meeting, the MACHO Project completed an analysis of a two year data set that included light curves for nine million stars. The new analysis differed from that described above and in [5] primarily in that new event acceptance criteria were developed following the project's experience in the Galactic bulge. Eight candidate microlens events passed the new acceptance criteria, of which six were of high quality. The best estimate of the Macho fraction in the Galactic halo increased in this new analysis from $\sim 20 \%$ to $\sim 40 \%$ (six events included) or $\sim 50 \%$ (all eight events included), but still with very large error bars.

\section{Bibliographic Notes}

Two new and very good reviews have recently been written, by Paczynski [23] and by Roulet and Mollerach [26]. These provide a sound and broad introduction to the field. It is useful, in addition, to read some of the key early works, especially [21], which got all of this going. Paczynski himself is 
often very modest about this paper, pointing out (correctly) that the phenomenology of the point mass gravitational lens had been known already for decades! What was new, and important, was the connection he made to the dark matter problem.

On the experimental side, it is definitely worthwhile to read the first announcements from MACHO [4], from EROS [7], and from OGLE [27]. The papers are very cautious, but nevertheless there is a sense of barely contained excitement that marks a discovery paper!

Now that the field is maturing, new directions suggest themselves. Three papers have generated considerable discussion. In the first, Gould [14] pointed out the great value that satellite based parallax measurements would bring to this area of research. This paper opened up the investigation of parallaxes and the more general study of novel ways to add to the information we gain from individual events. In the other two, Mao and Paczynski [20], and later Gould and Loeb [15] described gravitational microlensing by planetary systems; this may lead to a major new initiative to search for extra-solar planetary systems using gravitational microlensing.

Research into gravitational microlensing makes use of the World Wide Web, and this is a useful way to learn up to date information. In particular, the MACHO and OGLE projects make real time event information available this way, so their locations are given here. Current information on the MACHO Project may be found at: http://wwwmacho.mcmaster.ca/, with 
a mirror copy at: http://wwwmacho.anu.edu.au/. Buttons on these pages direct the browser to real time information on events in progress, and in addition there are pointers to the other major projects (e.g., EROS).

Information on the OGLE Project is at: http://www.astrouw.edu.pl, with a mirror copy at: http://www.astro.princeton.edu/ ogle/.

\section{References}

[1] Alard. C., et al. 1995, The Messenger, No. 80, 31.

[2] Albrow, M., et al. 1995, preprint.

[3] Alcock, C., et al. 1993, Nature, 365, 621.

[4] Alcock, C., et al. 1995, ApJ, 445, 133.

[5] Alcock, C., et al. 1995, Phys. Rev. Lett., 74, 2967.

[6] Alcock, C., et al. 1995, ApJ, 454, L125.

[7] Aubourg, E., et al. 1993, Nature, 365, 623.

[8] Bahcall, J̣. N., Flynn, C., Gould, A., \& Kirhakos, S. 1994, ApJ, 435, L51.

[9] Bouquet, A., Kaplan, J., Melchior, A., Giraud-Héraud, Y., \& Baillon, P. 1993, preprint, astro-ph/931209.

[10] Crotts, A., \& Tomaney, A. 1993, Columbia preprint. 
[11] Evans, N.W. 1993, MNRAS, 260, 191.

[12] Fich, M., \& Tremaine, S. 1991, ARA\&A, 29, 409.

[13] Frieman, J., \& Scoccimarro, R. 1994, ApJ, 431, L23.

[14] Gould, A. 1994, ApJ, 421, L75.

[15] Gould, A., \& Loeb, A. 1992, ApJ, 396, 104.

[16] Griest, K. 1991, ApJ, 366, 412.

[17] Griest, K., et al. 1991, ApJ, 372, L79.

[18] Han, C., \& Gould, A. 1994, preprint, astro-ph/9410052.

[19] Kiraga M., \& Paczynski, B. 1994, ApJ, 430, 101.

[20] Mao, S., \& Paczynski, B. 1991, ApJ, 374, L37.

[21] Paczynski, B. 1986, ApJ, 304, 1.

[22] Paczynski, B. 1991, ApJ, 371, L63.

[23] Paczynski, B. 1996, ARA\&A, 34, in press.

[24] Primack, J., Seckel, D., \& Sadoulet, B. 1993, Ann. Rev. Nuc. Part. Sci., 38,751 .

[25] Sackett, P., \& Gould, A. 1993, ApJ, 419, 648. 
[26] Roulet, E., \& Mollerach, S. 1996, Physics Reports, in press.

[27] Udalski, A., et al. 1993, Acta Astronomica, 43, 289.

[28] Udalski, A., et al. 1994, ApJ, 426, L69.

[29] Udalski, A., et al. 1994, Acta Astronomica, 44, 165.

[30] Zhao, H. S., Spergel, D. N., \& Rich, R. M. 1995, ApJ, 440, L13. 


\section{Figure Captions}

Figure 1: Two microlensing events seen by the MACHO Project, with the discovery date of the latter shown.

Figure 2: Lightcurve of an event from the MACHO Project showing the parallax effect. The dashed line is the best fit not including the motion of the earth around the sun, the solid line includes the motion of the earth around the sun.

Figure 3: Mass of Macho versus distance to the Macho, and likelihood estimates assuming the lens is dark (long dash) or a main sequence star (short dash). 


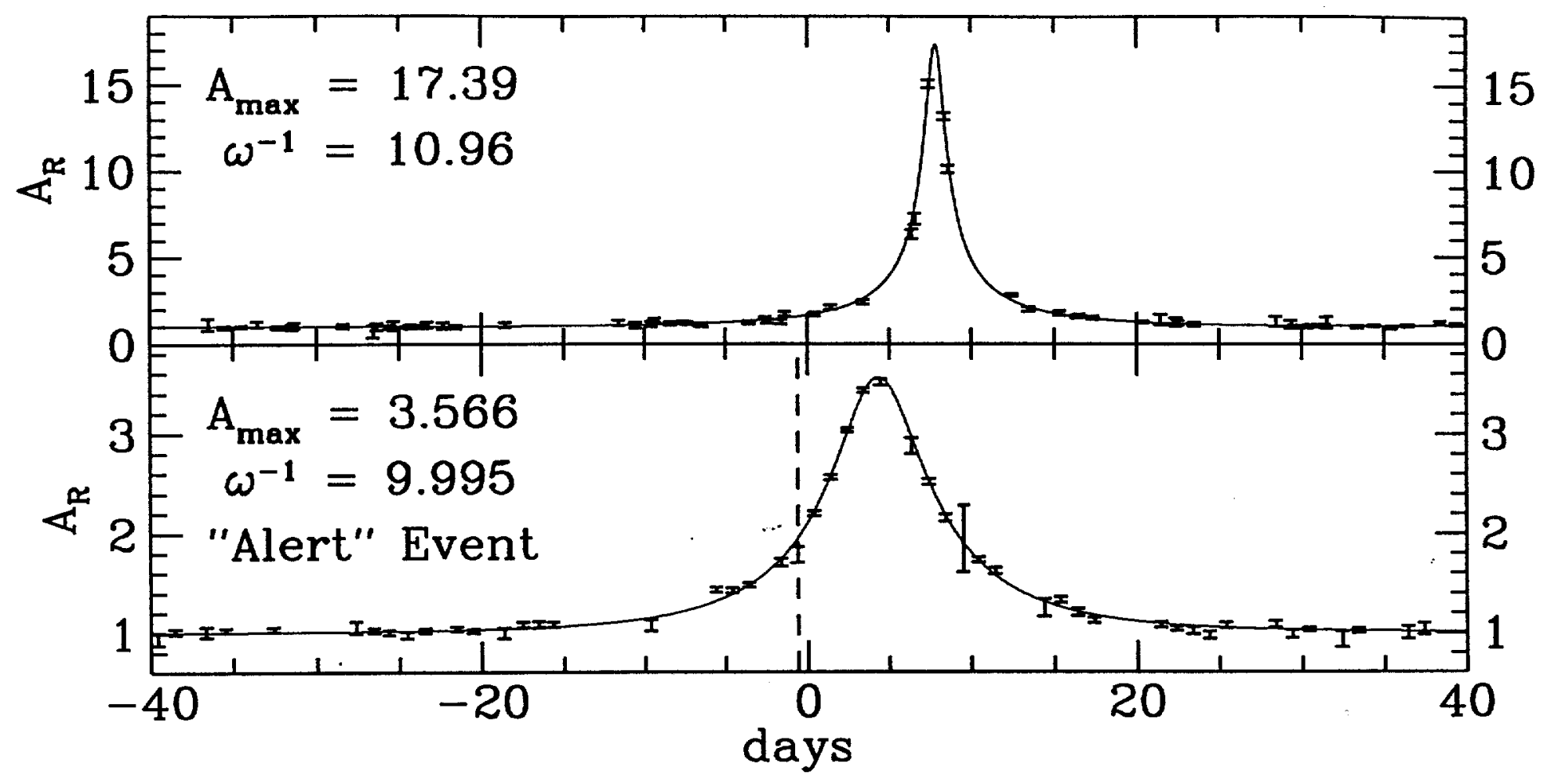




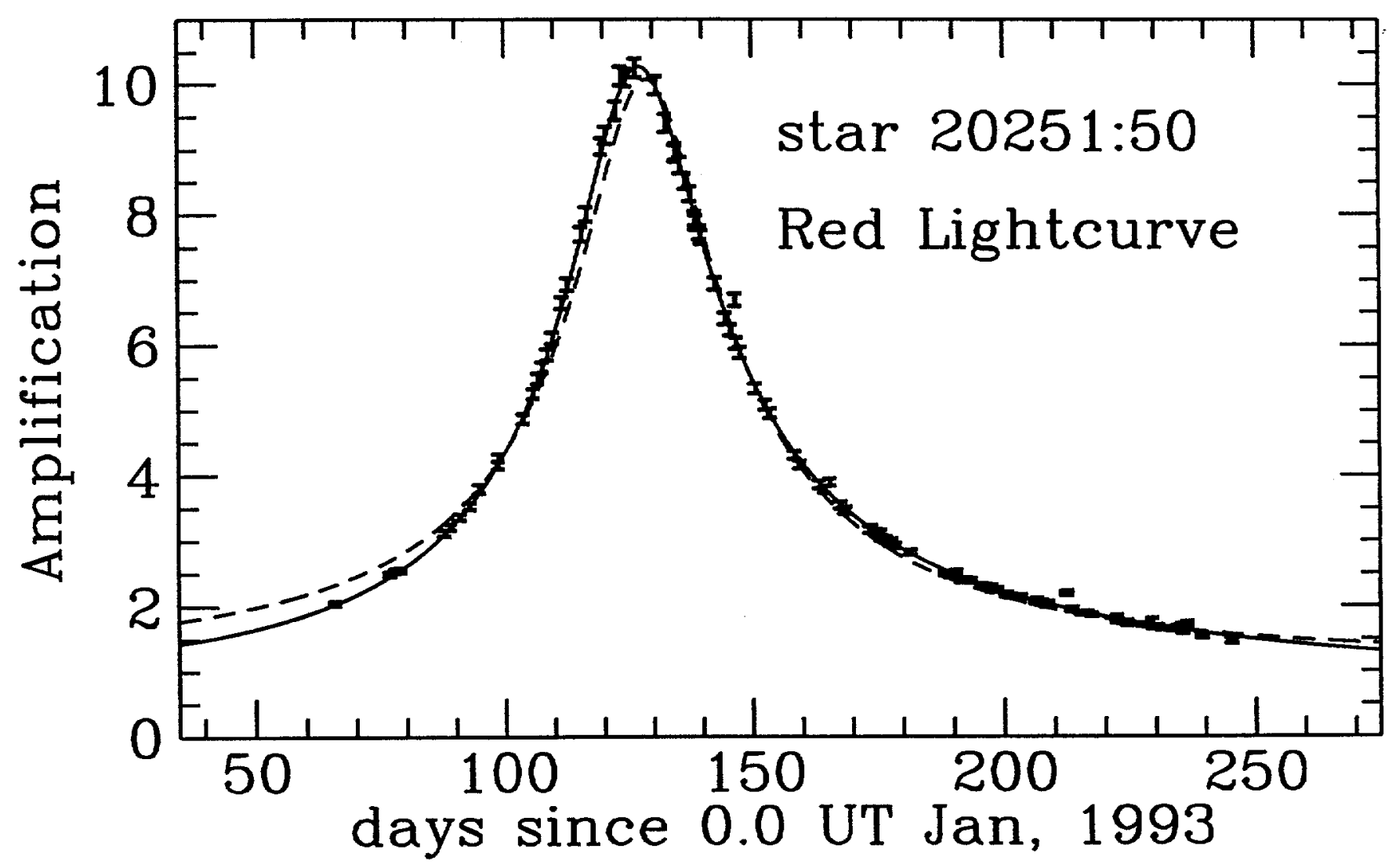









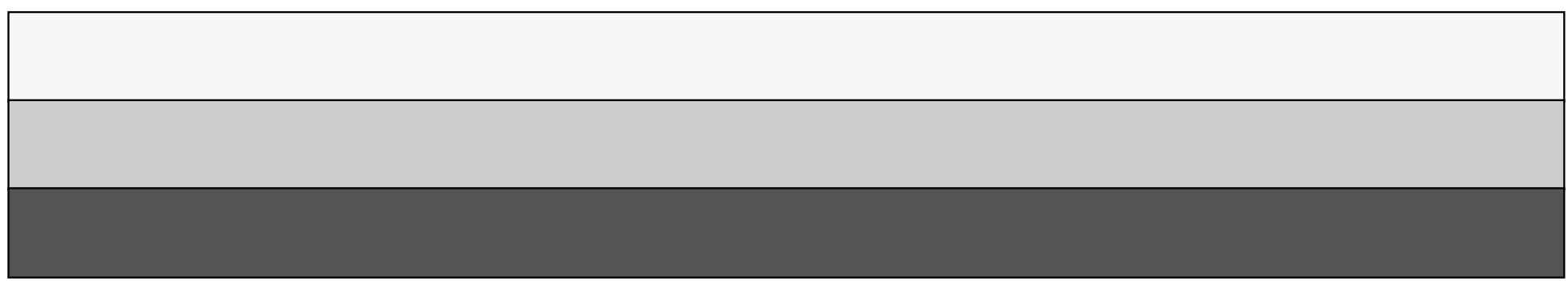

